\title{
Further developments of a fuzzy set map comparison approach
}

\author{
Alex Hagen-Zanker ${ }^{1}$, Bas Straatman ${ }^{2}$ and Inge Uljee ${ }^{1}$ \\ 1 \\ Research Institute for Knowledge Systems, P.O. Box 463, 6200 AL Maastricht, the \\ Netherlands \\ 2 \\ Memorial University of Newfoundland, Department of Geography, St. John's, \\ Newfoundland, Canada, A1B 3X9
}

Correspondence to Alex Hagen-Zanker

email: ahagen@riks.nl

Keywords: map comparison, fuzzy, similarity, Kappa, accuracy assessment, validation, calibration

\section{Acknowledgements}

Recent advances to the Map Comparison Kit have been made by order of the Dutch National Institute of Public Health and the Environment (RIVM). Earlier work on the Map Comparison Kit was possible as part of contracts for the Dutch National Institute for Coastal Zone and Marine Management (RIKZ) and the European Commission Joint Research Centre. 


\section{Further developments of a fuzzy set map comparison approach}

Abstract:

Fuzzy set map comparison offers a novel approach to map comparison (Hagen 2003). The approach is specifically aimed at categorical raster maps and applies fuzzy set techniques, accounting for fuzziness of location and fuzziness of category, to create a similarity map as well as an overall similarity statistic: the Fuzzy Kappa. To date, the calculation of the Fuzzy Kappa (or K-fuzzy) has not been formally derived and the documented procedure was only valid for cases without fuzziness of category. Furthermore, it required an infinitely large, edgeless map. This paper presents the full derivation of the Fuzzy Kappa; the method is now valid for comparisons considering fuzziness of both location and category and does not require further assumptions. This theoretical completion opens opportunities for use of the technique that surpass the original intentions. In particular, the categorical similarity matrix can be applied to highlight or disregard differences pertaining to selected categories or groups of categories and to distinguish between differences due to omission and commission. 


\section{Introduction}

The methods presented in this paper are in essence an extension to the methods presented in (Hagen 2003). That paper introduced a method for comparing categorical maps taking into account both proximity relations and the fact that some pairs of categories are more similar than others. Proximity relations are generally not taken into account in map comparison methods as most methods are based on analysis of the contingency table, which summarizes cell-to-cell agreement and disagreement (Foody 2002). The fuzzy set map comparison belongs to a less prominent, but growing, tradition of considering geographical coherence in the assessment of map similarity. Approaches that do so address the presence and overlap of features (Power et al. 2002, Remmel \& Perera 2002), local composition and configuration (Csillag \& Boots 2004), apply swap heuristics (Ehlschlager 2000, Fewster \& Buckland 2001) or compare maps that are rescaled to different resolutions (Costanza 1989, Pontius 2002). The approach in (Hagen 2003) is different all together, but takes most after the swap and multi-resolution methods, as differences found at a location may be mitigated by categories found in the neighbourhood.

The current paper has three main parts: in first instance the full derivation of the Fuzzy Kappa is introduced. This derivation fills the theoretical gap that remained in the original paper. Next the use of the categorical similarity matrix is discussed and applications of the matrix are detailed that were not originally intended or documented. The next section demonstrates the advances made, by re-examining the datasets of the original paper. All analysis presented in this paper is performed using the Map Comparison Kit software, which is freely available on the Internet (RIKS 2004).

\section{Full derivation of the Fuzzy Kappa statistic}

In a (crisp) categorical map each cell belongs to one category. In the fuzzy set map comparison, an interpretation of the map is made, indicating in the form of a vector how similar the cell is to 
each of the categories found on the other map. This vector is now called the interpretation vector. A cell can be similar to multiple categories at the same time and the sum of all its similarity values may be larger than 1 . Thus, if we consider similarity to be a degree of belonging, the interpretation vector is a fuzzy set (Zadeh, 1965).

The interpretation vector is based on two ideas, fuzziness of location and fuzziness of category. By fuzziness of location is meant that a cell is principally defined by the category found there, but to a lesser extent it is also defined by the categories found in its neighbourhood. This means that a cell is similar to the categories found in its proximity. By fuzziness of categories is meant that the distinction between some categories is not sharp and hence some categories are more akin to each other than others. Here the concept of fuzziness is stretched to mean similarity even though that is something else. For instance, the categories 'broad leaved forest' and 'pine forest' are sharply distinct, but for many purposes they can be considered similar.

As the interpretation vector is a fuzzy set, fuzzy set theory becomes available and we could use fuzzy similarity measures to express the agreement between the cells at one location in a pair of maps. For instance, a typical min-max similarity measure could be applied on the two interpretation vectors. This approach is not followed however, because it would introduce an unnecessary indirection, as by their definition the interpretation vectors already address similarity directly. (It would also be incorrect because the elements in the vectors refer to different categories.) Thus, by direct use of the interpretation vectors, two indications for local similarity are found; 1) the element of the interpretation vector of the location in the first map that refers to the category found in the second map and 2) its counterpart; the element of the interpretation vector of the location in the second map, that refers to the category found in the first map. These two indications of local similarity are combined into a single similarity value. For this, the fuzzy logic AND operation is used. Practically, this means that the local similarity the lesser of the two indications of local similarity. 
Besides similarity per cell, also an overall statistic of agreement is calculated. This statistic is called the Fuzzy Kappa, as its definition is analogous to the Kappa statistic. It gives the average similarity corrected for similarity to be expected given the total area taken in by each category and is based on probability and not fuzzy set theory. The motivation to discount for the expected similarity is to prevent the overall similarity statistic from bias towards maps with uneven frequency distributions. Another bias, which is not corrected for, is towards maps with fragmented landscapes. As maps are more fragmented, neighbourhoods become more diverse and thus it becomes more likely to find a 'mitigating' category in the neighbourhood. This has the consequence that Fuzzy Kappa values for pairs of maps that are highly clustered may be lower than intuitively expected, as reported by Wealands et al. (2004)

The calculation of average and expected similarity is given in the following two sections. Subsequently the calculation of the Fuzzy Kappa is detailed.

\subsection{Calculation of the overall similarity}

The raster maps to be compared are not necessarily rectangular and may also contain gaps. Moreover, the two maps that are to be compared $\left(\operatorname{map}^{A}\right.$ and $\left.m a p^{B}\right)$ do not need to cover the exact same area, and only for the area they both cover, similarity values are calculated. The nonoverlapping parts of the maps do play a role in the comparison, because these parts influence the neighbourhood configuration of cells that are being compared as well as the frequency distribution of categories over the maps.

Thus, we have two sets of locations (for $m a p^{A}$ and $m a p^{B}$ ) lying on a regular grid as expressed below:

$$
\begin{aligned}
& \operatorname{locs}^{A}=\left\{V_{1}^{A}, V_{2}^{A}, \cdots, V_{n^{A}}^{A}\right\} \\
& \operatorname{locs}^{B}=\left\{V_{1}^{B}, V_{2}^{B}, \cdots, V_{n^{B}}^{B}\right\} \\
& \text { locs }=\operatorname{locs}^{A} \cap \operatorname{locs}^{B}=\left\{V_{1}, V_{2}, \cdots, V_{n}\right\}
\end{aligned}
$$


where $V_{i} \in \mathbb{N}^{2}$ and $V_{i}^{A}=V^{B}=V_{i} \forall i \leq n$. This means that all cells are specified by a row and column number and that the locations are sorted to the effect that the first $n$ elements of $\operatorname{locs}^{A}$ and $\operatorname{locs}^{B}$ coincide. The third set of locations, locs, is the intersection of the former two. A local similarity will be calculated for all cells present in locs.

Every cell on map A and B is occupied by one of the categories present in their respective legends. Let $C^{A}$ and $C^{B}$ be the sets of categories present in the legends of $\operatorname{map}^{A}$ or $\operatorname{map}^{B}$. For the sake of notational simplicity the cell categories are considered identical to their index number in $C^{A}$ and $C^{B}$ :

$$
\begin{aligned}
& C^{A}=\{1,2, \ldots, r\} \\
& C^{B}=\{1,2, \ldots, s\}
\end{aligned}
$$

where $r$ and $s$ are the number of categories present in the legends of $\operatorname{map}^{A}$ and $m a p^{B}$.

The functions $m^{A}$ and $m^{B}$ (equation 3 ) read the category found respectively in map $^{A}$ and $m a p^{B}$ given a location. Thus, $m_{l}^{A}$ is the category found at location $l$ in $m a p^{A}$ :

$$
\begin{aligned}
& m^{A}: \operatorname{locs}^{A} \rightarrow C^{A} \\
& m^{B}: \operatorname{locs}^{B} \rightarrow C^{B}
\end{aligned}
$$

The comparison of the two maps at a cell is based upon the configuration of the neighbourhood of that cell in both maps. The neighbourhood of a cell consists of all cells within a certain distance from that cell, including the cell itself. The defining radius is constant over the maps, but not necessarily the same for both maps. The neighbourhood configuration of a cell consists of two vectors: Vector $N$ contains all the categories found in the neighbourhood. Vector $D$ contains the corresponding distances to the central cell of the neighbourhood. The relations are expressed in equations (4) and (5) below:

$$
\begin{aligned}
& N_{l}^{A}=\left\{n_{l, 1}^{A}, n_{l, 2}^{A}, \cdots, n_{l, t_{l}}^{A}\right\} \\
& N_{l}^{B}=\left\{n_{l, 1}^{B}, n_{l, 2}^{B}, \cdots, n_{l, u_{l}}^{B}\right\}
\end{aligned}
$$




$$
\begin{aligned}
& D_{l}^{A}=\left\{d_{l, 1}^{A}, d_{l, 2}^{A}, \cdots, d_{l, t_{l}}^{A}\right\} \\
& D_{l}^{B}=\left\{d_{l, 1}^{B}, d_{l, 2}^{B}, \cdots, d_{l, u_{l}}^{B}\right\}
\end{aligned}
$$

where $u_{l}$ and $t_{l}$ are the size of the neighbourhood for map A and B at location $l$. The size of the neighbourhood differs from location to location due to the edges of the map. The first cell in the neighbourhood is by definition the central cell, thus $d_{l, l}^{A}=d_{l, l}^{B}=0, n_{l, 1}^{A}=m_{l}^{A}$ and $n_{l, 1}^{B}=m_{l}^{B}$.

The influence of neighbouring locations diminishes with distance according to a function $F$ (equation 6). This function is not necessarily the same for both maps, but always returns the value 1 for the central cell and returns a value between 0 and 1 for all other neighbouring cells, as follows:

$$
\begin{aligned}
& F^{A}: \mathbb{R}^{+} \rightarrow[0,1] \subset \mathbb{R} \\
& F^{B}: \mathbb{R}^{+} \rightarrow[0,1] \subset \mathbb{R} \\
& F^{A}(0)=F^{B}(0)=1
\end{aligned}
$$

The comparison method also takes into account that some categories found in the legends of $m a p^{A}$ and $m a p^{B}$ are more similar to each other than others. This is expressed by an index of similarity between 0 and 1 for each combination of categories. Categorical similarity is thus expressed as matrix $M$ in equation 7 :

$$
M=\left(\begin{array}{ccc}
M_{1,1} & \cdots & M_{1, s} \\
\vdots & \ddots & \vdots \\
M_{r, 1} & \cdots & M_{r, s}
\end{array}\right)
$$

where $M_{i, j} \in[0,1] \subset \mathbb{R}$. The row-index of the matrix relates to the categories found in map $^{A}$ and the column index to those in $m a p^{B}$. Categorical similarities are assumed bi-directional, meaning that the similarity of category $a$ in $\operatorname{map}^{A}$ to category $b$ in $m a p^{B}$ is identical to that of category $b$ in $m a p^{B}$ to category $a$ in $m a p^{A}$ and its value is $M_{a, b}$.

For every location two interpretation vectors are calculated, $S_{l}^{A}$ and $S_{l}^{B}$. These vectors express for both maps how similar that location is to all categories found in the other map. For one category, 
this equals the maximum contribution to the similarity over all locations in the neighbourhood, taking into account both the categorical similarity and the distance decay function. Equation 8 formulizes this relation:

$$
\begin{aligned}
& S_{l}^{A}=\left\{\operatorname{sim}_{l, 1}^{A}, \operatorname{sim}_{l, 2}^{A}, \cdots, \operatorname{sim}_{l, s}^{A}\right\} \\
& S_{l}^{B}=\left\{\operatorname{sim}_{l, 1}^{B}, \operatorname{sim}_{l, 2}^{B}, \cdots, \operatorname{sim}_{l, r}^{B}\right\} \\
& \operatorname{sim}_{l, b}^{A}=\max _{j=1}^{t_{l}}\left(M_{n_{l, j}^{A}, b} \cdot F^{A}\left(d_{l, j}^{A}\right)\right) \\
& \operatorname{sim}_{l, a}^{B}==\max _{k=1}^{u_{l}}\left(M_{a, n_{l, k}^{B}} \cdot F^{B}\left(d_{l, k}^{B}\right)\right)
\end{aligned}
$$

where $a$ and $b$ are indices to $C^{A}$ respectively $C^{B}$.

Equation 9 calculates the overall similarity of the cell, $S_{l}$, by taking the minimum similarity of $\operatorname{map}^{A}$ to the category found in $m a p^{B}$ at that location and vice versa:

$$
S_{l}=\min \left(\operatorname{sim}_{l, m_{l}^{B}}^{A}, \operatorname{sim}_{l, m_{l}^{A}}^{B}\right)
$$

The map similarity is calculated as the average similarity over all cells, as in equation 10 :

$$
S=\frac{\sum_{l=1}^{n} S_{l}}{n}
$$

\subsection{Calculation of the expected overall similarity}

Equation 11 defines the probability of a cell on $\mathrm{map}^{A}$ to be taken in by category $a$ according to the frequency of occurrence of $a$ on A:

$$
p_{a}^{A} \equiv \frac{\sum_{l=1}^{n^{A}} \delta_{m_{l}^{A}, a}}{n^{A}}
$$

where $\delta_{x, y}=1$ when $x=y$ and 0 otherwise. The $p^{B}{ }_{b}$ 's are defined analogously.

Based on this definition of the probabilities regarding the category occupying a cell in $m a p^{A}$ and $m_{a p}{ }^{B}$, we can now calculate the expected value of $S$ for the comparison area.

The local similarity, as expressed in equation 9, only depends upon the neighbourhood configuration found in $m a p^{A}$ and $m a p^{B}$. Considering that the distance vectors $D_{l}$ are fixed, then 
the similarity only depends on the categories found at the different offsets in the neighbourhood. This means that, as the number of neighbourhood configurations is limited, the number of possible local similarity values is too. The vector $Z_{i}$ contains all possible neighbourhood occupancies for cell $l$ (equation 13). The number of possible combinations (z) follows from the number of cells in the neighbourhoods $\left(t_{l}\right.$ and $u_{l}$ ) and the number of categories present in the maps $(r$ and $s)$ as below:

$$
\begin{gathered}
z=r^{t_{l}} * s^{u_{l}} \\
Z_{l}=\left(N_{l, 1}, N_{l, 2}, \cdots, N_{l, z}\right) \\
N_{l, i}=\left(N_{l, i}^{A}, N_{l, i}^{B}\right) \\
N_{l, i}^{A}=\left(n_{l, i, 1}^{A}, n_{l, i, 2}^{A}, \cdots n_{l, i, t}^{A}\right) \\
N_{l, i}^{B}=\left(n_{l, i, 1}^{B}, n_{l, i, 2}^{B}, \cdots n_{l, i, u}^{B}\right)
\end{gathered}
$$

The vectors $P_{i}$ (equation 14) and $X_{i}$ (equation 15) give respectively the probability and local similarity value that correspond to each neighbourhood configuration.

$$
\begin{gathered}
P_{l}=\left(p_{l, 1}, p_{l, 2}, \cdots, p_{l, z}\right) \\
p_{l, i}=\prod_{j=1}^{t} p_{n_{l, i, j}^{A}}^{A} \cdot \prod_{k=1}^{u} p_{n_{l, i, k}^{B}}^{B} \\
X_{l}=\left(x_{l, 1}, x_{l, 2}, \cdots, x_{l, z}\right) \\
x_{l, i}=\min \left[\max _{j=1}^{t_{l}}\left(M_{n_{l, i, 1}^{A}, n_{l, i, j}^{B}} \cdot F^{A}\left(d_{l, j}^{A}\right)\right), \max _{k=1}^{u_{l}}\left(M_{n_{l, i, k}^{A}, n_{l, i, 1}^{B}} \cdot F^{B}\left(d_{l, k}^{B}\right)\right)\right]
\end{gathered}
$$

In other words, $P_{i}$ is the probability distribution of the outcome of the similarity values which are in the vector $X_{i}$, and thus the expected local similarity can be calculated as the sum product of probability and similarity (see equation 16$)$.

$$
E\left(S_{l}\right)=\sum_{i=1}^{z}\left(p_{l, i} \bullet x_{l, i}\right)
$$

Equation 17 calculates the expected similarity as the average expected similarity over all cells. 


$$
E=E(S)=\frac{\sum_{l=1}^{n} E\left(S_{l}\right)}{n}
$$

The number $z$ can be quite large and for many practical purposes the straightforward implementation of the equations presented here will not be possible. Substantial efficiency gains in the calculation can be made by taking opportunity of the fact that there are large groups of neighbourhood configurations that lead to an identical similarity value.

\subsection{Calculation of the Fuzzy Kappa}

The Fuzzy Kappa is calculated in the same manner as the (crisp) Kappa, as shown in equation 17:

$$
\text { FuzzyKappa }=K_{\text {Fuzzy }}=\frac{S-E}{1-E}
$$

The calculation detailed in this paper can be time consuming. An approximation can be made by assuming that all offsets found in the neighbourhood are present on the map for all locations. In that case the expected value of similarity is constant over the map and thus only needs to be calculated once. In practice this means that for equations 13 to 16 the subscript $l$ is cancelled and that $t=t^{\max }$ and $u=u^{\max }$.

\section{Extended application of the categorical similarity matrix}

Hagen (2003) proposed to use the categorical similarity matrix for the purpose of taking into account that some categories are more similar to each other than others. When, for instance, the categories 'pine forest' and 'broad leafed forest' are more similar to each other than to the categories 'urban' and 'agricultural land', then the matrix of table 1 may be applied. A second example considers categories of an ordinal nature; this example is given in table 2.

One new use of the category matrix is to temporarily set two or more categories equal. Thus the matrix functions as a tool for 'on the fly' reclassification. The similarity matrix of table 3 signifies that the difference between 'pine forest' and 'broad leafed forest' are ignored in the comparison. 
Another use of the categorical similarity matrix is to assess the similarity of a single category. Following Monserud \& Leemans (1992) all categories except the category being considered are set as being identical to each other. Table 4 gives the similarity matrix for this comparison regarding the category 'Urban'. Comparing maps per category can serve different purposes for instance it may be necessary to rank the different categories according to the degree of similarity in order to prioritize further actions. Knowing to what extent differences between maps are related to individual categories may also help to understand the nature of the differences.

Applying asymmetrical categorical similarity matrices gives the option to separately consider differences due to omission and commission or appearing and disappearing. The terms omission and commission have a meaning in the context of accuracy assessment, whereas appearing and disappearing relate to comparison of maps for different moments in time. Differences (errors) due to commission of a category are locations where the category is placed where it should not be (false positives) and differences due to omission are those where the category is not found, but where it should be (false negatives). In practical situations the implications of differences due to omission and commission may be quite different. For instance, models dedicated to the early detection of problem areas (e.g. fire, desertification, pollution) may, under the precautionary principle, be used with a high tolerance for errors due to commission and a small tolerance for errors due to omission. When used in a later stage (e.g. once resources are being allocated) this tolerance may be reversed. The similarity matrix given in table 5 is used to assess the fuzzy difference resulting from commission of the category urban (in $\mathrm{map}^{B}$ relative to $m a p^{A}$ ); this is achieved by considering only those cells dissimilar where 'Urban' is found in map $^{B}$ and not in $m a p^{A}$. The transposed matrix (table 6) registers differences due to omission instead.

An asymmetrical categorical similarity matrix can express differences in the weighting of omission and commission. The similarity matrix in table 7 gives such a matrix where omission is weighted stronger than commission. When exploring differences between two maps, such a 
setting would rarely have merit, because if omission and commission have a distinct meaning it is more worthwhile to consider them in two separate maps than to confound them in a single one. However, when applied in an automated procedure, such as the automatic calibration by Straatman et al. (2004) it is necessary to express similarity in a single statistic and better results may be obtained when different types of error are weighted differently.

The table may also be used to compare maps with unequal legends. The categorical similarity matrix can be either crisp (table 8) or fuzzy (table 9). Finding the appropriate correspondence between the two categorical definitions is not a straightforward task and is essentially subjective. Fritz and See (2004) developed a methodology to construct a categorical similarity matrix on the basis of a questionnaire filled out by experts who judge the similarity from different perspectives.

\section{Results}

To demonstrate the functioning of the different categorical similarity matrices, we apply them on the same datasets as Hagen (2003). The first data set is a synthetic one, constructed specifically to demonstrate the functioning of the fuzzy set map comparison.

Comparison of the maps in the synthetic dataset (figure 1) yields the result given in figure 2, where grey levels indicate similarity (as in all subsequent greyscale maps). In order to obtain a better understanding of the nature of the differences figures $3 \mathrm{a}$ to $3 \mathrm{~d}$ give an oversight of the

differences per category. Figures $3 \mathrm{e}$ to $3 \mathrm{~h}$ give the differences due to commission (in $\operatorname{map}^{B}$ relative to $m a p^{A}$ ) and figure $3 \mathrm{i}$ to 31 those due to omission. Table 10 gives the Fuzzy Kappa values resulting from the comparison, as well as regular Kappa values calculated according to Monserud \& Leemans (1992). It becomes clear that by considering proximity the order of the categories when sorted according to similarity changes. The similarity matrices that underlie the analysis for the whole map (figure 2) and for the category 'City' (figures 3b, 3f and 3j) are found in tables $11 \mathrm{a}$ to $11 \mathrm{~d}$. For the other categories the similarity matrices are analogous. 
The detailed similarity maps and statistics give information that cannot be derived directly from figure 2. In particular, we find that, according to table 10, the strongest contribution to the difference is made by the presence of 'River' in $\operatorname{map}^{B}$ where it is not present in map $^{A}$. Observing figure $3 \mathrm{k}$ it becomes clear that this is explained by the additional branch in the upper left area of the map. Furthermore, we learn that the maps are most similar with respect to the 'City' category. The similarity map of figure $3 \mathrm{~b}$ clarifies that for this category there is only one cell in the lowest class of similarity.

The second dataset (figure 4) is taken from practice. It consists of a land use map generated by a model and another which is considered 'ground truth'. The particular model is of the Constrained Cellular Automata (CCA) type (White et al. 1997) applied for the study of the urban development of Dublin, as part of the Moland project (White et al. 2000). Comparison of the two maps yields a similarity map (figure 5) and a Fuzzy Kappa of 0.905 which is considered satisfactory, because it means that the CCA model outperforms the null-model (Hagen 2003).

The influence of differences pertaining to the category 'Road and rail networks and associated land' is considered a disturbance in the comparison, because it signifies a difference in the maps that the model is not expected or intended to prevent; The CCA model takes note of roads and railways in the calculation of accessibility, but takes them as exogenous input from separate network layers and does not predict their development. To investigate the impact of the disturbance, the difference with respect to this category is considered by temporarily setting all other categories equal to each other. Maybe more significantly, the similarity remaining when ignoring this source of difference is calculated, by temporarily setting the category 'Road and rail...' equal to all other categories. The results (figure 6) indicate that dismissing the contribution of 'Road and rail...' has a distinct visual impact on the distribution of the differences because the dominant linear elements disappear from the similarity map. Thus, we have gained more insight into the structure of the original similarity map. Despite the strong visual impact, the overall 
statistics are hardly affected; at the three-digit accuracy supported by the software, the Fuzzy Kappa does not change.

The categories 'Residential discontinuous sparse urban fabric' and 'Industrial areas' are of particular interest, because the model is aimed at their development and these categories displayed a severe change over the model period. Therefore, these two categories are examined separately. Considering the category 'Residential...' (figure 7) it appears that the differences due to omission are more serious than those due to commission. This is an indication that new residential cells are too often placed close to existing ones (minor differences due to commission) and too few new clusters are generated (major differences due to omission). This confirms the notion that, from a modeller's perspective, correctly 'seeding' new urban areas is more difficult than 'growing' existing areas.

An automated procedure might take this into account by weighting differences due to commission less than those by omission. This would mean that the procedure 'prefers' the combination of large errors due to commission and small errors of omission over the opposite and would thus lead to parameters more aimed at 'seeding' than at 'growing'. Admittedly, this is a speculative preposition and future research will need to point out the merit of applying asymmetrical similarity matrices in this manner. Figure 8 gives the results of weighting omission respectively commission stronger, analogous to table 7 but with 0.8 as the intermediate value. It demonstrates that by using different weights, other areas are highlighted as being most dissimilar.

The observation with regards to 'Industrial areas' (figure 9) is similar to that of 'Residential...'; however an additional observation is made here. Specifically in the northern part of the map it appears that the spatial distribution of clusters of omission is similar to that of the clusters of commission, indicating that although cell-to-cell the maps are clearly different (even when applying a tolerance for small spatial differences), the model does capture significant aspects of the spatial structure of industrial location. 


\section{Conclusion}

Hagen (2003) offered a promising approach to the comparison of categorical maps taking into account fuzziness of location and fuzziness of category. The approach could be considered unfinished because it could not be readily applied to all cases for which it was intended. The current paper fills the theoretical gap, making it possible to calculate the Fuzzy Kappa for all cases.

Additionally it is pointed out that the similarity matrix has significance beyond the original intentions. Not only can the matrix be used to set similarities between categories; additionally, it can be used to single out or weigh categories or groups of categories in the comparison; In the evaluation of the land use model we disregard differences related to road and rail, not because this category is similar or identical to others, but instead because we consider this type of difference not relevant to our analysis.

Also the distinction between differences due to omission and commission can be investigated via the similarity matrix. Thus, the fuzzy set map comparison not only offers insight into the severity and spatial distribution of differences, but also the nature of these differences.

The similarity matrix offers a practically unlimited number of comparison settings. There is little point in calculating all of these every time a pair of maps is compared. Therefore, the aim of the method, as implemented in the Map Comparison Kit, is to illuminate differences and similarities found in a pair of maps through interactive, explorative use. It also means that although the methods are explicitly defined and repeatable, the idea of objective map comparison is a fiction. Comparison is based upon the subjective interpretation of maps, which is expressed firstly by the selection of the methodology and secondly by the parameter settings (if any) that are applied. 
The fact that in the second dataset we recognize structural similarity with regards to industrial location and clustering that is not reflected in the statistics makes the case for further research towards structure based map comparison.

\section{References}

Costanza, R., 1989, Model goodness of fit: A multiple resolution procedure, Ecological Modelling, 47, 199-215.

Ehlschlaeger, C.R., 2000, Representing uncertainty of area class maps with a correlated inter-map cell swapping heuristic, Computers, Environment and Urban Systems, 24, 451-469.

Fewster, R.M. \& Buckland, S.T., 2001, Similarity indices for spatial ecological data, Biometrics, 57, 495-501.

Foody, G.M., 2002, Status of land cover classification accuracy assessment, Remote Sensing of Environment, 80, 185-201.

Fritz, S. and See, L., 2004, Comparison of land cover maps using fuzzy agreement. Submitted to International Journal of Geographical Information Science

Hagen, A., 2003, Fuzzy set approach to assessing similarity of categorical maps. International Journal of Geographical Information Science, 17, 235-249

Monserud, R. A., and Leemans, R., 1992, Comparing global vegetation maps with the Kappa statistic. Ecological Modelling, 62, 275-293.

Pontius Jr., R.G., 2002, Statistical methods to partition effects of quantity and location during comparison of categorical maps at multiple resolutions, Photogrammetric Engineering and Remote Sensing, 68, 1041-1049. 
Power, C., Simms, A. \& White, R., 2001, Hierarchical fuzzy pattern matching for the regional comparison of land use maps, International Journal of Geographical Information Science, 15, 77-100.

Remmel, T.K. \& Perera, A.H., 2002, Accuracy of discontinuous binary surfaces: a case study using boreal forest fires, International Journal of Geographical Information Science, 16, 287298.

RIKS, 2004, Map Comparison Kit: product information on the Internet, http://www.riks.nl/products/Map_Comparison_Kit

Straatman, B., White, R. \& Engelen, G., 2004, Towards an automatic calibration procedure for constrained cellular automata, Computers, Environment and Urban Systems, 28, 149-170.

Wealands, S.R., Grayson, R.B. \& Walker, J.P., 2004, Quantitative comparison of spatial fields for hydrological model assessment--some promising approaches, Advances in Water Resources, In Press, Corrected Proof.

White, R., Engelen, G., and Uljee, I., 1997, The use of constrained cellular automata for highresolution modelling of urban land-use dynamics. Environment and Planning B: Planning and Design, 24, 323-343.

White, R., Engelen, G., Uljee, I., Lavalle, C. and Ehrlich, D., 2000, Developing an Urban Land use Simulator for European Cities. In Proceedings of the $5^{\text {th }}$ EC-GIS Workshop held in Stresa, Italy 38-30 June 1999, edited by E. Fullerton, (Ispra, Italy: European Commission, Joint Research Centre), 179-190.

Zadeh, L., 1965, Fuzzy sets, Information and Control, 8, 338-353. 


\section{TABLES}

\begin{tabular}{|l|c|c|c|c|}
\hline map A $\downarrow:$ map B $\rightarrow$ & pine... & broad... & urban & agri... \\
\hline pine forest & 1 & $\mathbf{0 . 5}$ & 0 & 0 \\
\hline broad leaved forest & $\mathbf{0 . 5}$ & 1 & 0 & 0 \\
\hline urban & 0 & 0 & 1 & 0 \\
\hline agricultural land & 0 & 0 & 0 & 1 \\
\hline
\end{tabular}

Table 1. An example similarity matrix, where pine and broad leaved forest are similar to each other.

\begin{tabular}{|l|c|c|c|c|c|}
\hline map A $\downarrow:$ map B $\rightarrow$ & high.... & medium.... & low... & agri... & forest \\
\hline high density residential & 1 & $\mathbf{0 . 4}$ & $\mathbf{0 . 2}$ & 0 & 0 \\
\hline medium density residential & $\mathbf{0 . 4}$ & 1 & $\mathbf{0 . 4}$ & 0 & 0 \\
\hline low density residential & $\mathbf{0 . 2}$ & $\mathbf{0 . 4}$ & 1 & 0 & 0 \\
\hline agriculture & 0 & 0 & 0 & 1 & 0 \\
\hline forest & 0 & 0 & 0 & 0 & 1 \\
\hline
\end{tabular}

Table 2. An example similarity matrix where, the residential categories have a ordinal relation

\begin{tabular}{|l|c|c|c|c|}
\hline map A $\downarrow:$ map B $\rightarrow$ & pine... & broad ... & urban & agri... \\
\hline pine forest & 1 & $\mathbf{1}$ & 0 & 0 \\
\hline broad leaved forest & $\mathbf{1}$ & 1 & 0 & 0 \\
\hline urban & 0 & 0 & 1 & 0 \\
\hline agricultural land & 0 & 0 & 0 & 1 \\
\hline
\end{tabular}

Table 3. An example similarity matrix, where pine and broad leaved forest are considered equal in the comparison

\begin{tabular}{|l|c|c|c|c|}
\hline map A $\downarrow:$ map B $\rightarrow$ & pine... & broad... & urban & agri... \\
\hline pine forest & 1 & 1 & $\mathbf{0}$ & 1 \\
\hline broad leaved forest & 1 & 1 & $\mathbf{0}$ & 1 \\
\hline urban & $\mathbf{0}$ & $\mathbf{0}$ & $\mathbf{1}$ & $\mathbf{0}$ \\
\hline agricultural land & 1 & 1 & $\mathbf{0}$ & 1 \\
\hline
\end{tabular}

Table 4. An example similarity matrix, where the category urban is considered separately.

\begin{tabular}{|l|c|c|c|c|}
\hline map A $\downarrow:$ map B $\rightarrow$ & pine... & broad ... & urban & agri... \\
\hline pine forest & 1 & 1 & $\mathbf{0}$ & 1 \\
\hline broad leaved forest & 1 & 1 & $\mathbf{0}$ & 1 \\
\hline urban & 1 & 1 & $\mathbf{1}$ & 1 \\
\hline agricultural land & 1 & 1 & $\mathbf{0}$ & 1 \\
\hline
\end{tabular}

Table 5. An example similarity matrix, where only commission of the category urban is considered 


\begin{tabular}{|l|c|c|c|c|}
\hline map A $\downarrow:$ map B $\rightarrow$ & pine... & broad... & urban & agri... \\
\hline pine forest & 1 & 1 & 1 & 1 \\
\hline broad leaved forest & 1 & 1 & 1 & 1 \\
\hline urban & $\mathbf{0}$ & $\mathbf{0}$ & $\mathbf{1}$ & $\mathbf{0}$ \\
\hline agricultural land & 1 & 1 & 1 & 1 \\
\hline
\end{tabular}

Table 6. Example similarity matrix, where only omission of the category urban is considered

\begin{tabular}{|l|c|c|c|c|}
\hline map A $\downarrow:$ map B $\rightarrow$ & pine... & broad... & urban & agri... \\
\hline pine forest & 1 & 1 & $\mathbf{0 . 5}$ & 1 \\
\hline broad leaved forest & 1 & 1 & $\mathbf{0 . 5}$ & 1 \\
\hline urban & $\mathbf{0}$ & $\mathbf{0}$ & $\mathbf{1}$ & $\mathbf{0}$ \\
\hline agricultural land & 1 & 1 & $\mathbf{0 . 5}$ & 1 \\
\hline
\end{tabular}

Table 7. An example similarity matrix, where only the category urban is considered and omission weights stronger than commission

\begin{tabular}{|l|c|c|c|c|c|}
\hline $\begin{array}{l}\text { map A } \downarrow: \\
\text { map B } \rightarrow\end{array}$ & $\begin{array}{l}\text { high dens. } \\
\text { residential }\end{array}$ & $\begin{array}{l}\text { medium dens. } \\
\text { residential }\end{array}$ & $\begin{array}{l}\text { low dens. } \\
\text { residential }\end{array}$ & agriculture & forest \\
\hline pine forest & 0 & 0 & 0 & 0 & $\mathbf{1}$ \\
\hline $\begin{array}{l}\text { broad leaved } \\
\text { forest }\end{array}$ & 0 & 0 & 0 & 0 & $\mathbf{1}$ \\
\hline urban & $\mathbf{1}$ & $\mathbf{1}$ & $\mathbf{1}$ & 0 & 0 \\
\hline $\begin{array}{l}\text { agricultural } \\
\text { land }\end{array}$ & 0 & 0 & 0 & $\mathbf{1}$ & 0 \\
\hline
\end{tabular}

Table 8. An example similarity matrix for the comparison of two maps with nonidentical legends and a crisp translation key

\begin{tabular}{|l|c|c|c|c|c|}
\hline $\begin{array}{l}\text { map A } \downarrow \text { : map } \\
\mathrm{B} \rightarrow\end{array}$ & $\begin{array}{l}\text { high dens. } \\
\text { residential }\end{array}$ & $\begin{array}{l}\text { medium dens. } \\
\text { residential }\end{array}$ & $\begin{array}{l}\text { low dens. } \\
\text { residential }\end{array}$ & agriculture & forest \\
\hline pine forest & 0 & 0 & 0 & $\mathbf{0 . 3}$ & 1 \\
\hline $\begin{array}{l}\text { broad leaved } \\
\text { forest }\end{array}$ & 0 & 0 & 0 & 0 & 1 \\
\hline urban & 1 & 1 & 1 & 0 & 0 \\
\hline $\begin{array}{l}\text { agricultural } \\
\text { land }\end{array}$ & 0 & 0 & $\mathbf{0 . 5}$ & 1 & 0 \\
\hline
\end{tabular}

Table 9. An example similarity matrix, for the comparison of two maps with nonidentical legends and a fuzzy translation key. In this example the distinction between 'low density residential' and 'agricultural land' cannot always be made and the definition of 'pine forest' in map A partially overlaps 'agriculture' in map B.

\begin{tabular}{|c|c|c|c|c|}
\hline $\mathrm{A} \downarrow \mathrm{B} \rightarrow$ & $\begin{array}{c}\text { Overall similarity } \\
\text { (Fuzzy Kappa) }\end{array}$ & $\begin{array}{c}\text { Disappearance } \\
\text { (Fuzzy Kappa) }\end{array}$ & $\begin{array}{c}\text { Appearance } \\
\text { (Fuzzy Kappa) }\end{array}$ & $\begin{array}{c}\text { Overall similarity } \\
\text { (Kappa) }\end{array}$ \\
\hline Open & 0.366 & 0.355 & 0.379 & 0.380 \\
\hline City & 0.616 & 0.592 & 0.644 & 0.556 \\
\hline River & 0.399 & 0.461 & $\mathbf{0 . 3 4 4}$ & 0.332 \\
\hline Park & 0.485 & 0.544 & 0.446 & 0.184 \\
\hline
\end{tabular}

Table 10. Per category comparison results 


\begin{tabular}{|c|c|c|c|c|}
\hline $\mathrm{A} \downarrow \mathrm{B} \rightarrow$ & Open & City & River & Park \\
\hline Open & 1 & 0 & 0 & 0 \\
\hline City & 0 & 1 & 0 & 0 \\
\hline River & 0 & 0 & 1 & 0 \\
\hline Park & 0 & 0 & 0 & 1 \\
\hline
\end{tabular}

Table 11a. Similarity matrix underlying results in figure 2

\begin{tabular}{|c|c|c|c|c|}
\hline $\mathrm{A} \downarrow \mathrm{B} \rightarrow$ & Open & City & River & Park \\
\hline Open & 1 & 1 & 1 & 1 \\
\hline City & 0 & 1 & 0 & 0 \\
\hline River & 1 & 1 & 1 & 1 \\
\hline Park & 1 & 1 & 1 & 1 \\
\hline
\end{tabular}

Table 11c. Similarity matrix underlying results in figure $3 f$

\begin{tabular}{|c|c|c|c|c|}
\hline $\mathrm{A} \downarrow \mathrm{B} \rightarrow$ & Open & City & River & Park \\
\hline Open & 1 & 0 & 1 & 1 \\
\hline City & 0 & 1 & 0 & 0 \\
\hline River & 1 & 0 & 1 & 1 \\
\hline Park & 1 & 0 & 1 & 1 \\
\hline
\end{tabular}

Table 11b. Similarity matrix underlying results in figure $3 b$

\begin{tabular}{|c|c|c|c|c|}
\hline $\mathrm{A} \downarrow \mathrm{B} \rightarrow$ & Open & City & River & Park \\
\hline Open & 1 & 0 & 1 & 1 \\
\hline City & 1 & 1 & 1 & 1 \\
\hline River & 1 & 0 & 1 & 1 \\
\hline Park & 1 & 0 & 1 & 1 \\
\hline
\end{tabular}

Table 11d. Similarity matrix underlying results in figure $3 j$ 


\section{COLOR FIGURES}

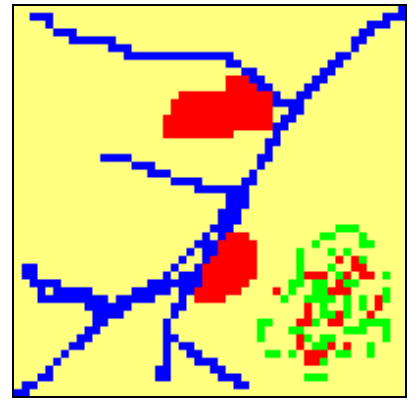

Figure la

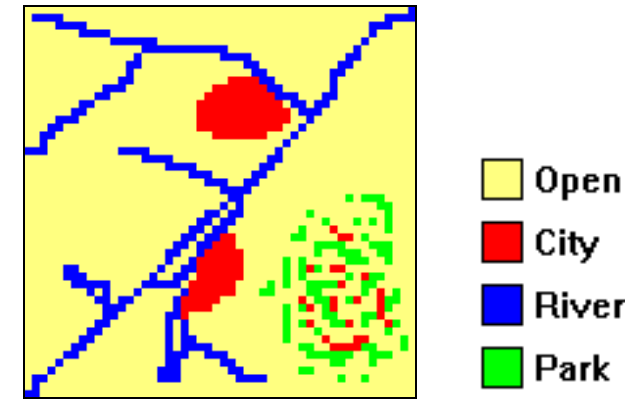

Figure $1 b$

Figure 1. Synthetic dataset

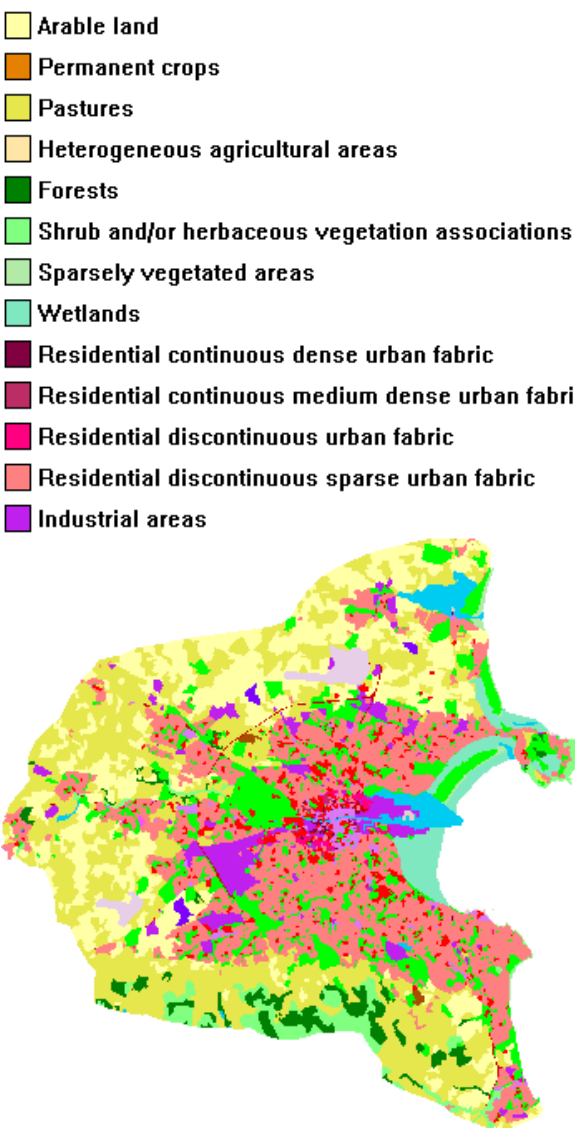

Figure $4 a$

$\square$ Commercial areas
$\square$ Public and private services
$\square$ Port areas
$\square$ Construction sites
$\square$ Abandoned area
$\square$ Road and rail networks and associated land
$\square$ Airport
$\square$ Mineral extraction sites
$\square$ Dump sites
$\square$ Artificial non-agricultural vegetated areas
$\square$ Water bodies
$\square$ Outside metropolitan area

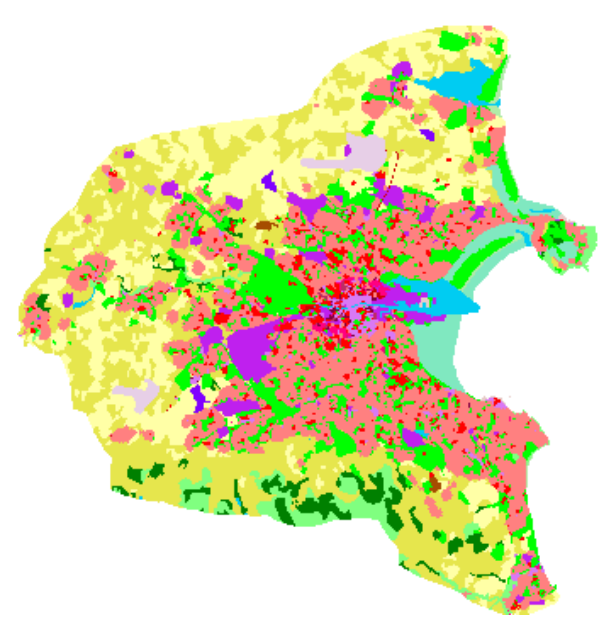

Figure $4 b$

Figure 4a. Ground truth map of Dublin in 1998

Figure 4b. Simulated map of Dublin in 1998 


\section{BW FIGURES}

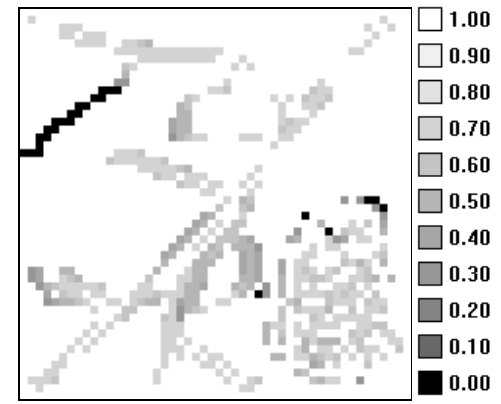

Figure 2. Fuzzy similarity, grey levels indicate local similarity, Fuzzy Kappa = 0.495

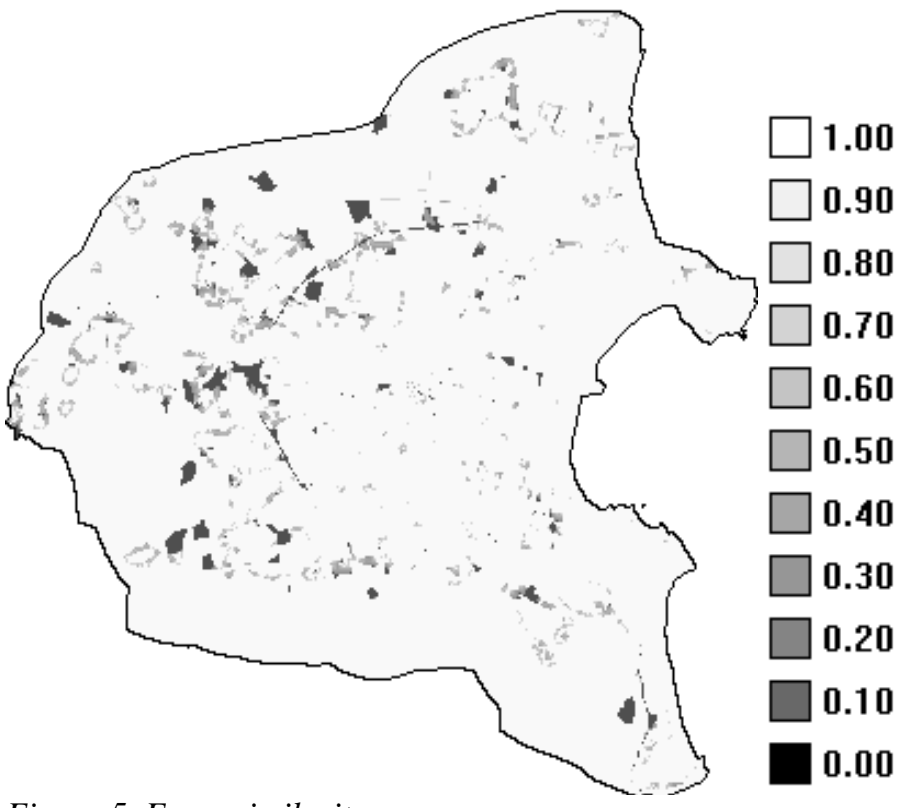

Figure 5. Fuzzy similarity 


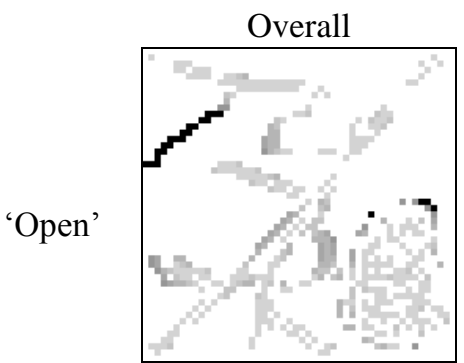

Figure $3 a$

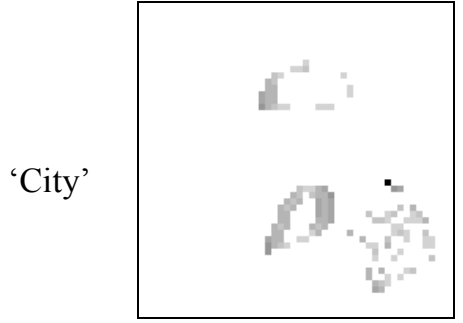

Figure $3 b$



Figure 3c

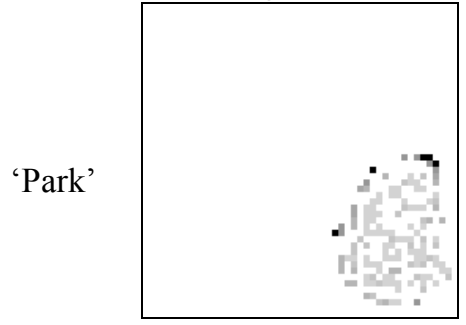

Figure 3d
Disappearance



Figure $3 e$



Figure $3 f$

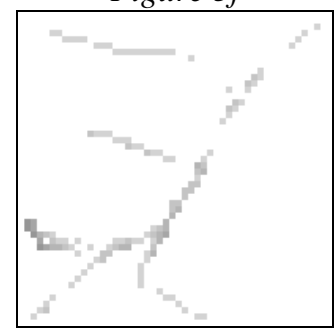

Figure $3 g$



Figure $3 h$

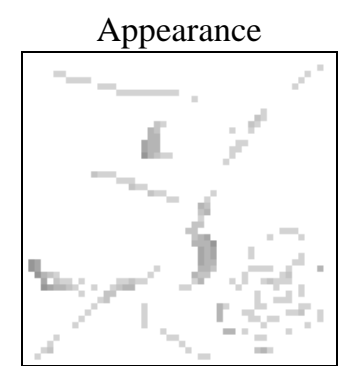

Figure $3 i$

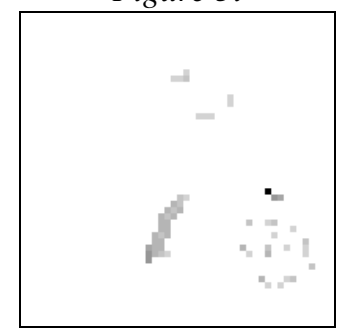

Figure 3j



Figure $3 k$

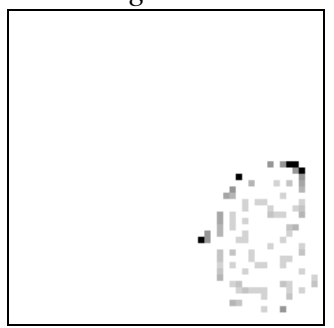

Figure $3 l$

Figures 3 Disagreement per category and split into disagreement due to appearance (omission) and disappearance (commission). 


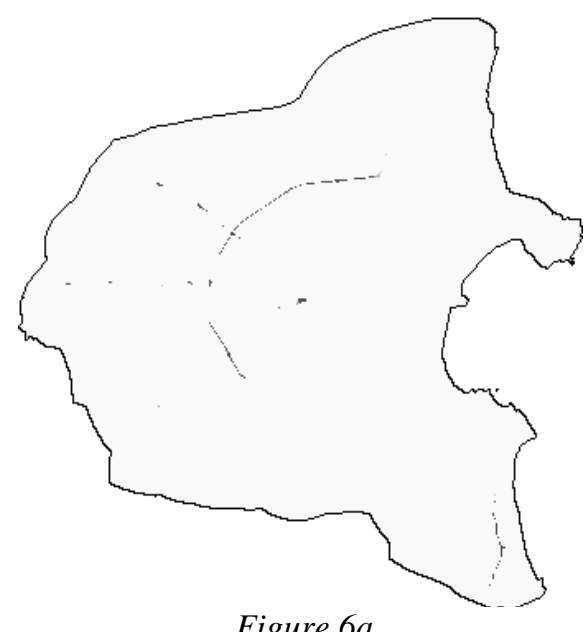

Figure $6 a$

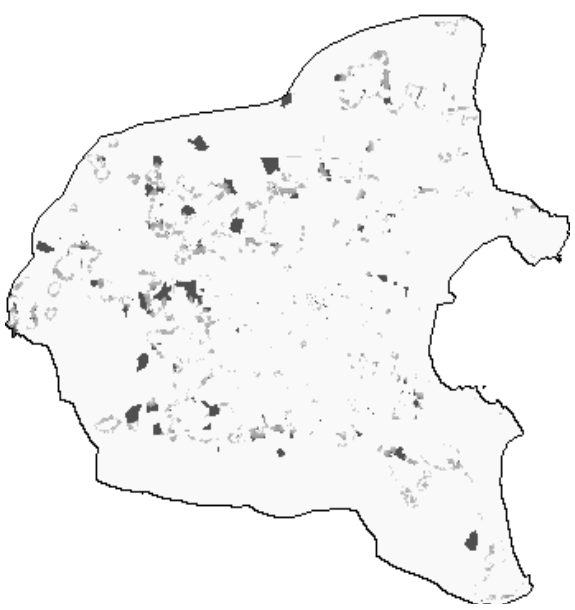

Figure $6 b$

Figure 6a. Fuzzy similarity of 'Road and rail networks and associated land' Figure 6b. Fuzzy similarity ignoring 'Roads and rail networks and associated land'

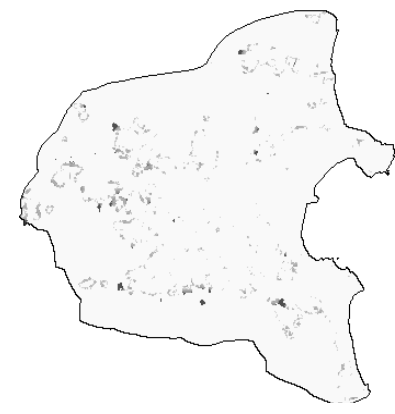

Figure $7 a$



Figure $7 b$

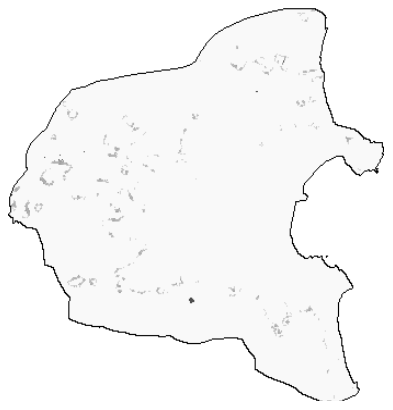

Figure $7 c$

Figure 7. Difference with respect to the category 'Residential discontinuous sparse urban fabric'. Overall (a), Omission (b) and Commission (c)

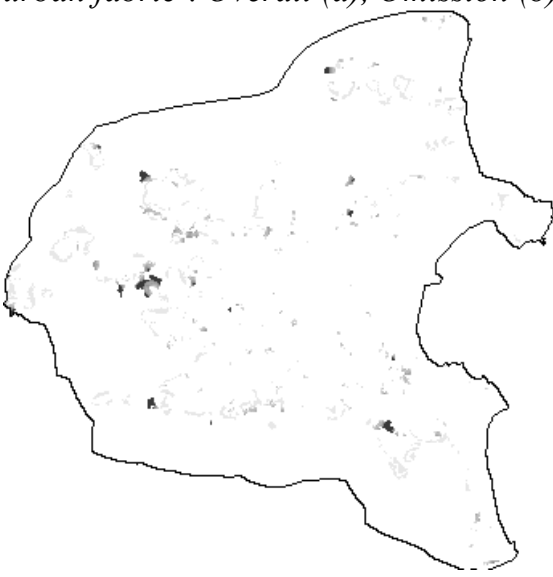

Figure $8 a$

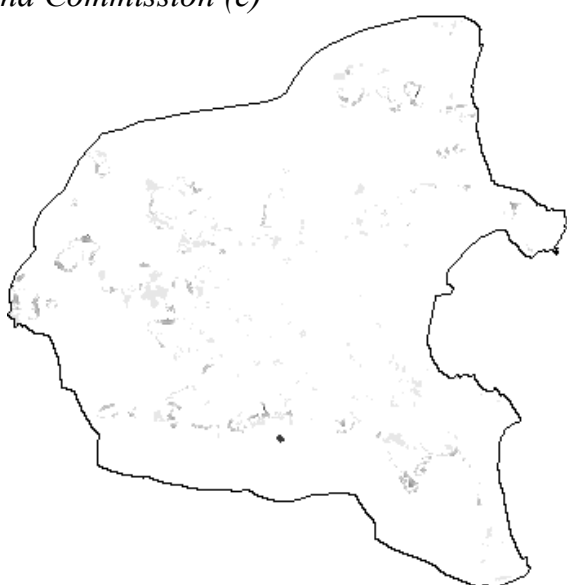

Figure $8 b$

Figure 8. Difference with respect to the category 'Residential discontinuous sparse urban fabric', where omission (a) respectively commission (b) is weighted stronger. 


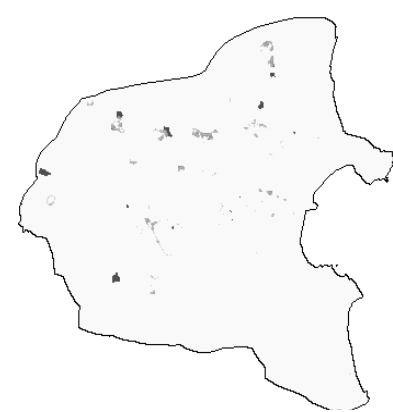

Figure $9 a$

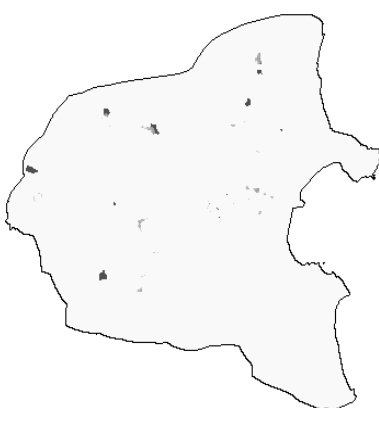

Figure $9 b$

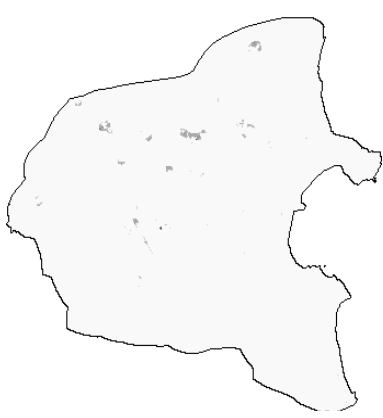

Figure 9c

Figure 9. Difference with respect to category 'Industrial areas'. Overall (a),

Omission (b) and Commission (c) 